\title{
Multiple hemorrhagic duodenal ulcers caused by cytomegalovirus infection
}

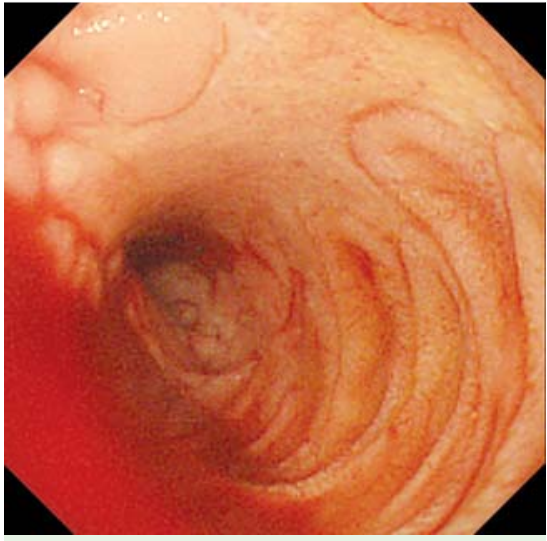

Fig. 1 Duodenum (third part): shallow, geographical ulcers.

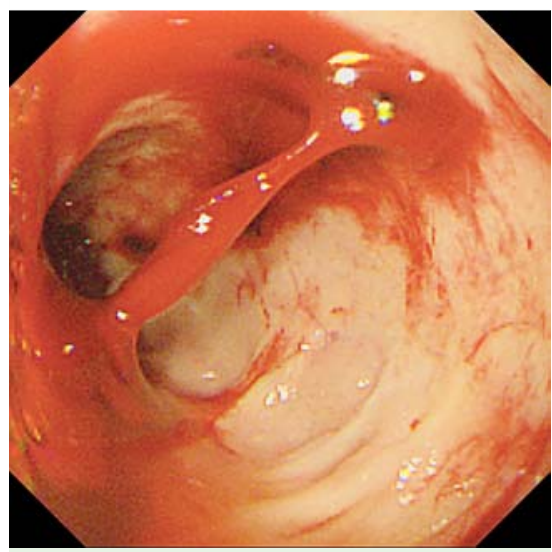

Fig. 2 Ulcer containing an exposed vessel that was bleeding heavily.

A 73-year-old man was hospitalized for cerebral trauma. While in the hospital, he passed tarry stool and developed anemia. He then suddenly had severe hemorrhage and went into shock. Emergent upper gastrointestinal tract endoscopy revealed several shallow, geographical ulcers with coagula in the second and third parts of the duodenum ( Fig. 1). One of the ulcers had an exposed vessel that was bleeding heavily ( Fig. 2). We stopped the bleeding by administering hypertonic saline with epinephrine and clipping the vessel. The next day, more exposed vessels were detected, and new bleeding points ap-
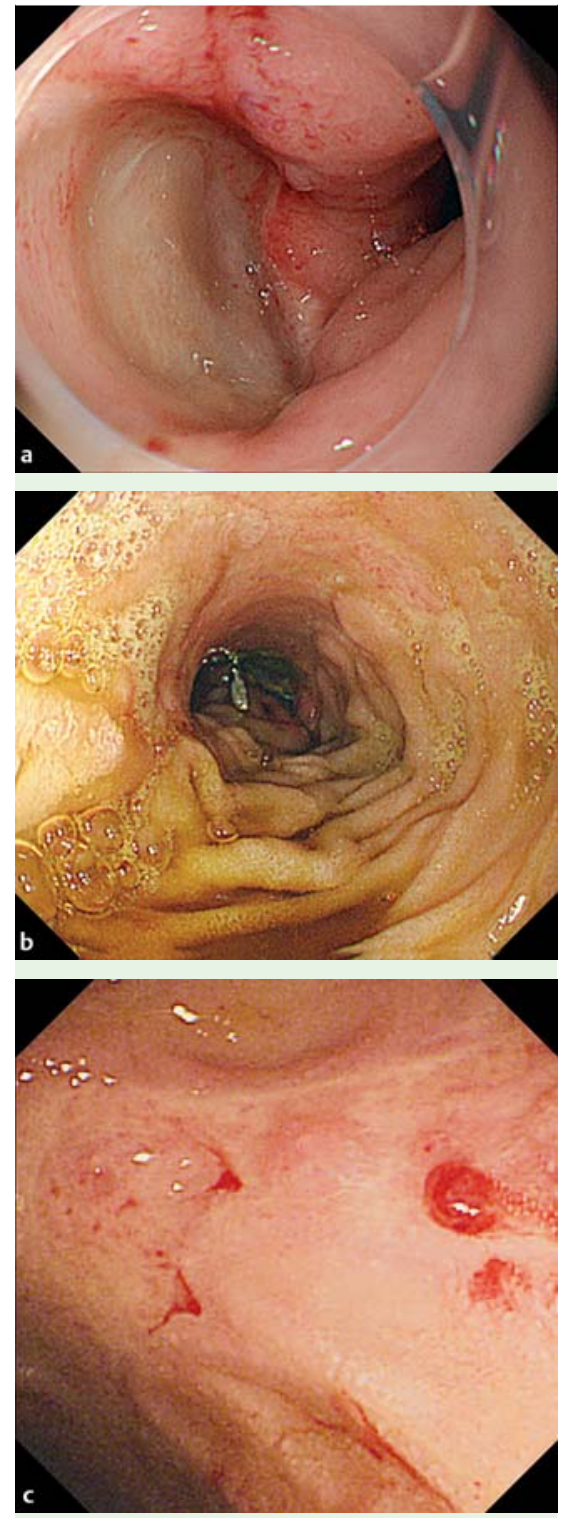

Fig. 3 A new punched-out ulcer. No exposed vessel is seen. $\mathbf{b}$ The ulcers became increasingly deeper daily. c New exposed vessels.

peared. The hemorrhage reoccurred and the ulcers grew larger and deeper every day. New punched-out ulcers were also detected $(\mathbf{3 a - c})$. The patient tested positive for serum cytomegalovirus (CMV) antigenemia and ganciclovir was administered. Although we attempted en-

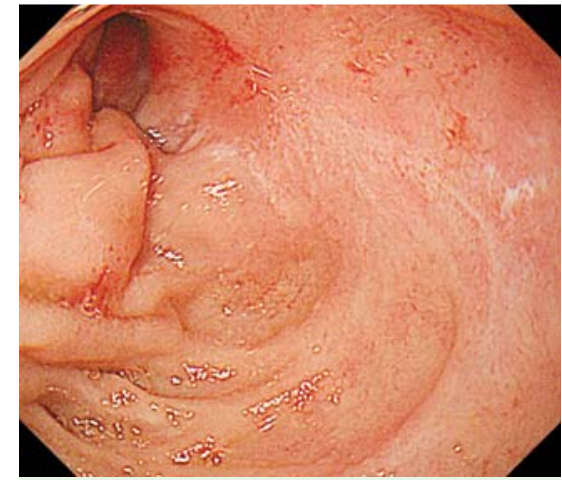

Fig. 4 Scarring of the ulcers following surgery and ganciclovir treatment.

doscopic hemostasis, we could not stop the hemorrhage completely, and the patient underwent surgery. Nuclear inclusion bodies were observed in the resected specimens. The patient was diagnosed as having hemorrhagic duodenal ulcers caused by CMV infection, and treatment with ganciclovir was continued. Following surgery and treatment with ganciclovir, scarring developed in the ulcers ( Fig. 4), and the patient tested negative for serum CMV antigenemia. Since then, the patient has not passed tarry stool and there has been no progression of anemia. He is likely to develop CMV-related disease because of his compromised status and major lesions in the stomach and colon caused by the CMV infection [1].

A search of the literature indicated that CMV lesions in the duodenum are rare, and hemorrhagic duodenal ulcers because of CMV infection are even more rare [2,3]. Ulcers related to CMV infection present in a variety of forms, for example, punched out, geographical, circular, longitudinal, etc. [4]. When a compromised host has bleeding in the alimentary tract and an ulcer with an atypical shape, CMV infection should be included in the differential diagnosis.

Endoscopy_UCTN_Code_CCL_1AB_2AZ_3AC 
R. Moroi, Y. Sato, T. Sakurai, S. Kin, T. Iwabuchi, M. Oyauchi, T. Igarashi, N. Obana

Division of Gastroenterology, Osaki Citizen Hospital, Furukawasennjuji, Osaki, Japan

\section{References}

1 Nagashima Y, Iida M, Hirakawa K et al. Gastrointestinal cytomegalovirus infection. Stomach and Intestine 2002; 37: 475-480

2 Wilcox CM, Schwartz DA. Symptomatic CMV duodenitis. An important clinical problem in AIDS. J Clin Gastroenterol 1992; 14: $293-297$

3 Yohei S, Naoe G, Shinji N et al. A case of acute gastroduodenitis with cytomegalovirus infection in a healthy adult. J Gastroenterol 2007; 104: $1383-1386$

4 Kiyotaka O, Wataru U, Koji S et al. Cytomegalovirus enterocolitis. Stomach and Intestine 2008; 43: 1653-1662

\section{Bibliography}

DOI $10.1055 / \mathrm{s}-0029-1214966$

Endoscopy 2009; 41: E216 - E217

(c) Georg Thieme Verlag KG Stuttgart · New York . ISSN 0013-726X

\section{Corresponding author}

R. Moroi, MD

Division of Gastroenterology

Osaki Citizen Hospital

2-3-10 Furukawasenjyuji

Osaki 989-6183

Japan

Fax: +81-229-235380

rinta@yb4.so-net.ne.jp 